\title{
Quantum Resonances in Chaotic Scattering
}

\author{
Kevin K. Lin* \\ Mathematics Department \\ Lawrence Berkeley National Lab \\ Berkeley, California 94720
}

\author{
Maciej Zworski ${ }^{\dagger}$ \\ Department of Mathematics \\ University of California \\ Berkeley, California 94720
}

\begin{abstract}
This letter summarizes numerical results from [1] which show that in quantum systems with chaotic classical dynamics, the number of scattering resonances near an energy $E$ scales like $\hbar^{-\frac{D\left(K_{E}\right)+1}{2}}$. Here, $K_{E}$ denotes the subset of the classical energy surface $\{H=E\}$ which stays bounded for all time under the flow of $H$ and $D\left(K_{E}\right)$ denotes its fractal dimension. Since the number of bound states in a quantum system with $n$ degrees of freedom scales like $\hbar^{-n}$, this suggests that the quantity $\frac{D\left(K_{E}\right)+1}{2}$ represents the effective number of degrees of freedom in chaotic scattering problems.
\end{abstract}

\section{Introduction}

Eigenvalues play a central role in the study of quantum bound states. In scattering, when the underlying classical system allows escape to infinity, resonances replace eigenvalues as fundamental quantities. One interpretation is that they correspond to metastable states. Physically, infinity models initial and final states in chemical reactions. The distribution of resonances in the case of chaotic scattering has been discussed in many places - see [2] for earlier work and [3] for recent references. In particular, we refer to [2] for a discussion of the physical motivation for this investigation.

Here, we present evidence that, in the case of hyperbolic classical dynamics, the density of quantum resonances is directly related to the dimension of the classical trapped set in the semiclassical limit. In the quantum setting, the density of states is important as it appears in semiclassical formulæ for reaction rates [4]. In classical dynamics, and for hyperbolic flows with "large in-

\footnotetext{
*E-mail address: kkylin@math.berkeley.edu

† E-mail address: zworski@math.berkeley.edu
}

finities," it was noted long ago [5] that the flow can be reduced to a fractal trapped set, forming "chaotic dust" after all else escaped. Our quantitative link between the two can be considered a manifestation of quantum chaos.

As far as metastable states in the interaction region are concerned, the effective number of degrees of freedom is given by the dimension of the trapped set:

$$
\begin{gathered}
\text { effective number of } \\
\text { degrees of freedom for } \\
\text { metastable states }
\end{gathered}=\frac{D\left(K_{E}\right)+1}{2} .
$$

More precisely, a classical result on the density of bound states (or resonances in strongly trapped regimes) says that the number $N_{\text {eig }}\left(E_{0}, E_{1}, \hbar\right)$ of eigenvalues in $\left[E_{0}, E_{1}\right]$ satisfies

$$
N_{\text {eig }}\left(E_{0}, E_{1}, \hbar\right) \approx \frac{\operatorname{vol}\left(\left\{E_{0} \leq H \leq E_{1}\right\}\right)}{(2 \pi \hbar)^{n}},
$$

where $n$ denotes the number of degrees of freedom, $H$ the classical Hamiltonian, and vol (.) phase space volume. This result is the Weyl law, and it has no known generalizations to resonances.

However, a conjecture based on the work of Sjöstrand [6] and Zworski [7] states that the number of resonances $\lambda=E-i \gamma$ with $E_{0}<E<E_{1}$ and $0<\gamma<\hbar$ behaves like

$$
\hbar^{-\frac{D\left(K_{E}\right)+1}{2}}, \hbar \rightarrow 0,
$$

where

$$
\begin{aligned}
D(\cdot) & =\text { dimension, } \\
K_{E} & =K \cap\{H=E\}, \\
K & =\left\{(x, p): \sup _{t}\left\|\Phi_{t}(x, p)\right\|<\infty\right\}, \\
\Phi_{t} & =\text { flow generated by } H .
\end{aligned}
$$

This letter summarizes numerical evidence [1] supporting the conjecture. 


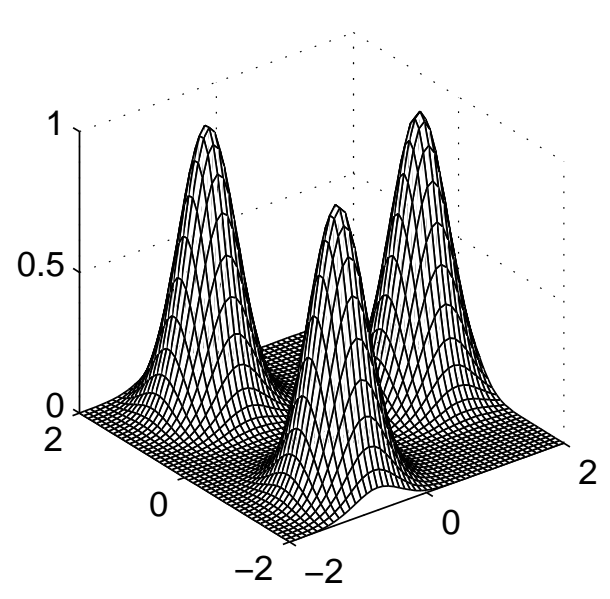

Figure 1: Triple gaussian potential

The computations there concern the Hamiltonian $H=(1 / 2)\left(p_{x}^{2}+p_{y}^{2}\right)+V(x, y)$, where the potential

$$
V(x, y)=\sum_{k=0}^{2} e^{-\frac{\left(x-x_{k}\right)^{2}+\left(y-y_{k}\right)^{2}}{2 \sigma^{2}}}
$$

consists of three gaussians placed symmetrically around the origin at a distance $R$ :

$$
\begin{aligned}
& x_{k}=R \cos \left(\frac{2 \pi k}{3}\right), \\
& y_{k}=R \sin \left(\frac{2 \pi k}{3}\right) .
\end{aligned}
$$

Quantizing $H$ yields the operator $\widehat{H}$

$$
-\frac{\hbar^{2}}{2}\left(\frac{\partial^{2}}{\partial x^{2}}+\frac{\partial^{2}}{\partial y^{2}}\right)+V(x, y)
$$

See Figure 1.

Remarks. The sets $K$ and $K_{E}$ are trapped sets and consist of initial conditions which generate trajectories that remain bounded for all time. The model considered in [1] has very few trapped trajectories, and $K$ and $K_{E}$ have vanishing Lebesgue measures. In fact, they are fractal. Also, in this paper, the term "chaotic" always means hyperbolic; see [8], [6].

\section{Generalizing the Weyl Law}

One can heuristically derive the conjecture as follows: First, note that a metastable state corresponding to a resonance $\lambda=E-i \gamma$ has a time-dependent factor of the form $e^{-\frac{i}{\hbar} \lambda t}=$ $e^{-\frac{i}{\hbar} E t} \cdot e^{-\frac{\gamma}{\hbar} t}$. A wave packet whose dynamics is dominated by $\lambda$ (and other resonances near it) would therefore exhibit temporal oscillations of frequency $O(E / \hbar)$ and lifetime $O(\hbar / \gamma)$. Heuristically, then, the number of times the particle "bounces" in the trapping region before escaping should be comparable to $\frac{E}{\hbar} \cdot \frac{\hbar}{\gamma}=\frac{E}{\gamma}$.

As $\hbar \rightarrow 0$, the dynamics of the wave packet should be well-approximated by classical mechanics. Let $T\left(x, y, p_{x}, p_{y}\right)$ denote the time for the particle to escape the system starting at position $(x, y)$ with momentum $\left(p_{x}, p_{y}\right)$. The trapping region has diameter $O(R)$, and typical velocities in the energy surface $\{H=E\}$ are $O(\sqrt{E})$ (mass is set to unity), so the number of times a classical particle bounces before escaping should be $O(T \sqrt{E} / R)$. This suggests that, in the limit $\hbar \rightarrow 0, T \sqrt{E} / R \sim$ $E / \gamma$ and consequently

$$
T \sim \frac{R \sqrt{E}}{\gamma} .
$$

Fix $\gamma_{0}>0$, and consider

$$
N_{\text {res }}=\#\left\{E-i \gamma: E_{0} \leq E \leq E_{1}, \gamma \leq \gamma_{0}\right\}
$$

for fixed energies $E_{0}$ and $E_{1}$ : Equation (8) implies that $T \geq R \sqrt{E_{0}} / \gamma_{0}$, so by analogy with the Weyl law,

$$
\frac{\operatorname{vol}\left(\left\{E_{0} \leq H \leq E_{1}, T \geq \frac{R \sqrt{E_{0}}}{\gamma_{0}}\right\}\right)}{(2 \pi \hbar)^{n}}
$$

follows as an approximation for the number of quantum states with the specified energies and decay rates.

Now, the function $1 / T$ is nonnegative and vanishes on $K_{\left[E_{0}, E_{1}\right]}=K \cap\left\{E_{0} \leq H \leq E_{1}\right\}$. Assuming that $1 / T$ is sufficiently regular, it should vanish quadratically on $K_{\left[E_{0}, E_{1}\right]}$. This suggests

$$
1 / T\left(x, y, p_{x}, p_{y}\right) \sim d_{K_{\left[E_{0}, E_{1}\right]}}\left(x, y, p_{x}, p_{y}\right)^{2},
$$

where $d_{K_{\left[E_{0}, E_{1}\right]}}$ denotes distance to $K_{\left[E_{0}, E_{1}\right]}$. It follows that $N_{\text {res }}$ should scale like

$$
\frac{\operatorname{vol}\left(\left\{E_{0} \leq H \leq E_{1}, d_{K_{\left[E_{0}, E_{1}\right]}} \leq \gamma_{0}^{\frac{1}{2}}\right\}\right)}{\hbar^{n}} .
$$

For small $\gamma_{0}$, this becomes

$$
C\left(R, E_{0}, E_{1}\right) \cdot \hbar^{-n} \cdot \gamma_{0}^{\frac{2 n-D\left(K_{\left[E_{0}, E_{1}\right]}\right)}{2}}
$$


for some constant $C$, by the definition of Minkowski dimension. Choosing $\gamma_{0}=\hbar$ and assuming that $\left|E_{1}-E_{0}\right|$ is small enough that $D\left(K_{E}\right)$ is approximately constant on $\left[E_{0}, E_{1}\right]$, we obtain

$$
N_{\text {res }} \sim \hbar^{-\frac{D\left(K_{E}\right)+1}{2}}
$$

In [6], Sjöstrand proved the following rigorous upper bound: For $\gamma_{0}>0$ satisfying $C \hbar<\gamma_{0}<$ $1 / C$,

$$
N_{\text {res }}=O\left(C_{\delta} \hbar^{-n} \gamma_{0}^{\frac{2 n-D\left(K_{\left[E_{0}, E_{1}\right]}\right)+\delta}{2}}\right)
$$

holds for all $\delta>0$. When the trapped set is of pure dimension, we can take $\delta=0$. Setting $\gamma_{0}=\hbar$ gives an upper bound of the form (14).

Sjöstrand's proof uses a semiclassical argument involving escape functions and the Weyl inequality for singular values. It motivates our heuristic discussion here.

In [7], a similar result for scattering on convex co-compact hyperbolic surfaces with no cusps was proved. Moving to a geometric setting was motivated by the availability of a large class of examples with hyperbolic flows, easily computable dimensions, and the hope that the Selberg trace formula could help obtain lower bounds. But, these hopes remain unfulfilled so far [9], and that partly motivates this work.

\section{Numerical Results}

The resonance computation uses complex scaling (see [10] for the origins of the method, and [11] for recent mathematical treatments and references), which associates to $\widehat{H}$ a family $\widehat{H}_{\alpha}$ of "scaled" operator. Each $\widehat{H}_{\alpha}$ can be discretized and its eigenvalues computed, as described in [1]. Those eigenvalues of $\hat{H}_{\alpha}$ which remain invariant under small perturbations in $\alpha$ are resonances. This is the strength of this fairly expensive method: We have a way of verifying the computation by varying the parameter.

As an illustration of complex scaling, Figures 2 and 3 contain resonances for $R=1.4$ and $\hbar=0.017$ and $\hbar=0.025$, respectively. Eigenvalues for different values of $\alpha$ are marked by different styles of points, and the box has depth $\hbar$ and width 0.2 , with $E_{0}=0.4$ and $E_{1}=0.6$. These plots may seem somewhat empty because only those eigenvalues near the region of interest

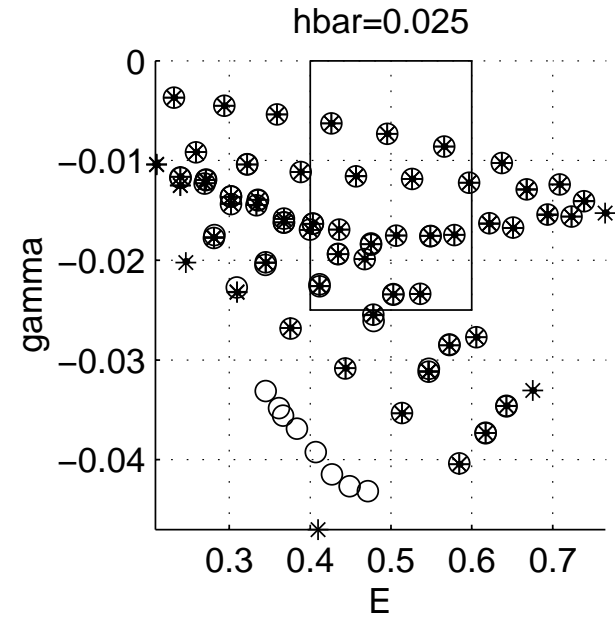

Figure 2: Eigenvalues for $E=0.5, \hbar=0.025$, $R=1.4$, and $\alpha \in\{0.0624,0.0799,0.0973\}$.

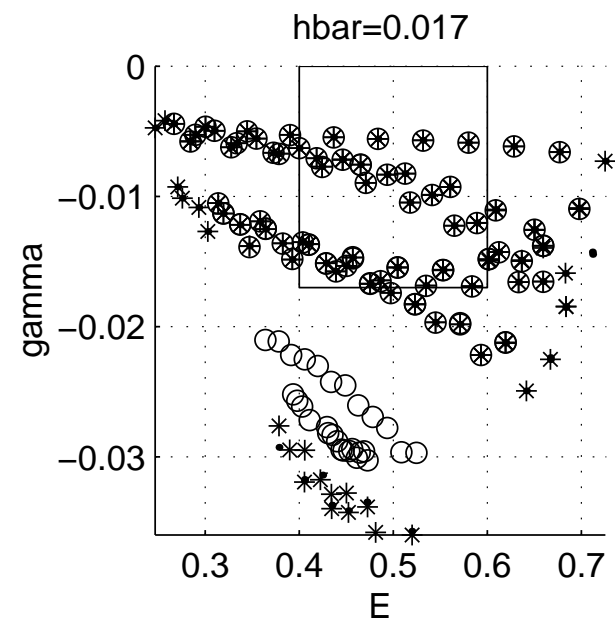

Figure 3: Eigenvalues for $E=0.5, \hbar=0.017$, $R=1.4$, and $\alpha \in\{0.0425,0.0599,0.0774\}$. 


\section{$\mathrm{R}=1.45$}

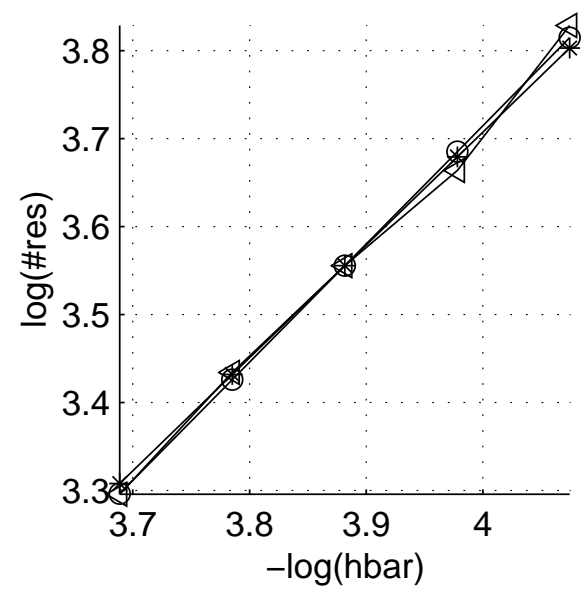

Figure 4: A plot of $\log \left(N_{\text {res }}\right)$ as a function for $-\log (\hbar)$ for $R=1.45$. The value of $\hbar$ ranges from 0.025 to 0.017 . Triangles represent numerical data, circles least squares regression, and stars the slope predicted by the conjecture.

\begin{tabular}{|c|c|c|c|}
\hline$R$ & slope & $\frac{D\left(K_{E}\right)+1}{2}$ & relative error \\
\hline \hline 1.4 & 1.2475 & 1.2885 & 0.032888 \\
\hline 1.45 & 1.3433 & 1.2834 & 0.044645 \\
\hline 1.5 & 1.2822 & 1.2829 & 0.00052244 \\
\hline 1.55 & 1.327 & 1.2773 & 0.037472 \\
\hline 1.6 & 1.3055 & 1.2725 & 0.025256 \\
\hline 1.65 & 1.2304 & 1.2694 & 0.031756 \\
\hline 1.7 & 1.2431 & 1.2636 & 0.016509 \\
\hline
\end{tabular}

Table 1: This table shows the slopes extracted from resonance data, as well as $\frac{D\left(K_{0.5}\right)+1}{2}$. Relative errors are also shown.

were computed. Notice the cluster of eigenvalues near the bottom edge of the plots: These are not resonances because they vary under perturbations in $\alpha$. Instead, they belong to an approximation of the (scaled) continuous spectrum.

Computed values of $\log \left(N_{\text {res }}\right)$ show that it is roughly a linear function of $-\log (\hbar)$, as one can see in Figures 4 and 5. We extract the slopes using least squares regression; these are shown in Table 1.

For comparison, $D\left(K_{E}\right)$ can be estimated using Poincaré sections. These are shown in Table 2 for various values of $R$. It is important to note that, while the dimension does depend on $E$ and $R$, it only does so weakly: Relative to its value, $D\left(K_{E}\right)$ is very roughly constant across the range

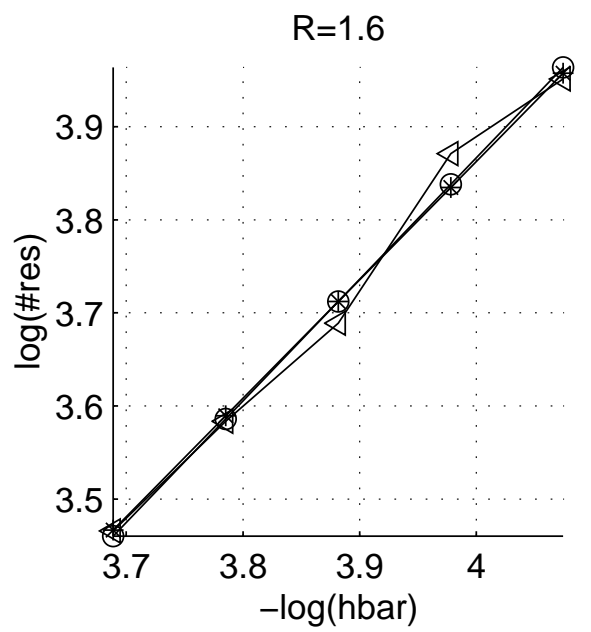

Figure 5: A plot of $\log \left(N_{\text {res }}\right)$ as a function for $-\log (\hbar)$ for $R=1.6$. The value of $\hbar$ ranges from 0.025 to 0.017 . The least squares regression is also shown.

\begin{tabular}{|c|c|c|c|}
\hline$R$ & $E=0.4$ & $E=0.5$ & $E=0.6$ \\
\hline \hline 1.4 & 1.3092 & 1.2885 & 1.261 \\
\hline 1.45 & 1.3084 & 1.2834 & 1.2558 \\
\hline 1.5 & 1.3037 & 1.2829 & 1.2497 \\
\hline 1.55 & 1.3007 & 1.2773 & 1.2521 \\
\hline 1.6 & 1.2986 & 1.2725 & 1.2511 \\
\hline 1.65 & 1.2912 & 1.2694 & 1.2488 \\
\hline 1.7 & 1.2893 & 1.2636 & 1.2524 \\
\hline
\end{tabular}

Table 2: This table contains estimates of $\frac{D\left(K_{E}\right)+1}{2}$ as a function of $R$. 
of $R$ and $E$ computed.

\section{Discussion}

Table 1 compares $\frac{D\left(K_{0.5}\right)+1}{2}$ as a function of $R$, versus the scaling exponents extracted from the resonance computation. Even though there are some fluctuations, the agreement with the conjectured law (3) is good. At the present time, the source of the noise is not known, but it is possibly due to the fact that the range of $\hbar$ explored here is simply too large to exhibit the asymptotic behavior clearly. It is also possible that the trapping time does not vanish quadratically everywhere on the trapped set, which would produce variations in the scaling law.

Aside from verifying the conjecture, the data also hints at more detailed structures in the distribution of resonances: In Figures 2 and 3, one can clearly see gaps and strips in the distribution of resonances. While we do not have rigorous error bounds for the dimension estimates, the numerical results are convincing.

\section{Acknowledgments}

We would like to thank Bill Miller for helpful comments on the first version of this letter.

KL was supported by the Fannie and John Hertz Foundation. MZ was partly supported by the NSF under grant DMS-9970614.

\section{References}

[1] K. Lin, U. C. Berkeley CPAM preprint PAM-790 (2001) math. SP / 0105026.

[2] P. Gaspard, S. A. Rice, J. Chem. Phys. 90 (1989), 2242; J. Chem. Phys. 90 (1989), 2255.

[3] A. Wirzba, Phys. Rep. 309 (1999), no. 1-2 chao-dyn/9712015.

[4] W. H. Miller, Chem. Rev. 87 (1987), 19-27.

[5] D. Sullivan, Publ. IHES 50 (1979), 172202.

[6] J. Sjöstrand, Duke Math. J. 60 (1990), 1-57.

[7] M. Zworski, Invent. Math. 136 (1999), 353409.
[8] P. Gaspard, S. A. Rice, J. Chem. Phys. 90 (1989), 2225.

[9] L. Guillopé, M. Zworski, Geom. and Func. Anal. 6 (1999), 1156-1168.

[10] J. Aguilar, J. M. Combes, Comm. Math. Phys. 22 (1971), 269-279; E. Balslev, J. M. Combes, Comm. Math. Phys. 22 (1971), 280-294.

[11] J. Sjöstrand, M. Zworski, J. Amer. Math. Soc. 4 (1991), 729-769; A. LahmarBenbernou, A. Martinez, Math. Phys. Archive preprint mp_arc 01-104. 